\title{
Disseminated necrotizing leukoencephalopathy eight months after alemtuzumab treatment for multiple sclerosis
}

\author{
Imke Metz ${ }^{1 *} \mathbb{D}$, Peter Rieckmann², Boris-Alexander Kallmann ${ }^{3}$ and Wolfgang Brück
}

\section{To the editor}

We report a case of disseminated necrotizing leukoencephalopathy (DNL) occurring after alemtuzumab treatment for multiple sclerosis (MS). A 33 year-old female patient with a 14-year history of highly active, relapsingremitting MS despite treatment with interferons, natalizumab and fingolimod was administered $12 \mathrm{mg}$ of alemtuzumab for five consecutive days. Alemtuzumab is a humanized monoclonal antibody directed against the CD52 antigen that significantly reduces relapses, MRI activity and the rate of sustained accumulation of disability [1]. Autoimmune disorders such as autoimmune thyroid disease occur in up to $28 \%$ of patients after alemtuzumab therapy and are associated with the phase of immune reconstitution [2, 3]. In addition, respiratory infections are well known side effects observed in $60 \%$ of patients [2]. Eight months after treatment, during a phase of clinically stable MS, the patient was admitted to the hospital due to anemia with low hemoglobin which was observed in her for the first time, and warm autoimmune hemolytic anemia was diagnosed. The hemoglobin dropped as low as $2.4 \mathrm{~g} / \mathrm{dl}$, requiring therapy with corticosteroids, intravenous immunoglobulins, plasma separation, cyclophosphamide and erythrocyte substitution. The anemia could not be stabilized. A lower respiratory tract infection with consecutive septic shock developed. The patient lost consciousness and required intubation. No focal neurological deficits were documented, and due to the fulminant disease course no MRI was performed. The patient died six days after admission and a brain autopsy was performed.

\footnotetext{
* Correspondence: imetz@gwdg.de

1 Department of Neuropathology, University Medical Center Göttingen, Georg-August-University, Robert-Koch-Str. 40, 37075 Göttingen, Germany Full list of author information is available at the end of the article
}

Autopsy revealed, besides characteristic inactive MS lesions, numerous small, round, disseminated and necrotizing lesions in the supratentorial white matter, the cerebellum and the brain stem including the pons. These lesions were characterized by numerous axonal spheroids and a pronounced macrophage infiltrate in the absence of lymphocytes, consistent with DNL (Fig. 1) $[4,5]$.

Severe autoimmune hemolytic anemia has been reported after alemtuzumab therapy, and may be caused by an immune dysregulation with the development of autoimmunity following drug application [6, 7]. Paradoxically, alemtuzumab is also used to treat autoimmune hemolytic anemia $[6,8]$.

The etiology of DNL is still unknown. It was first described in patients with brain tumors treated with highdose, methotrexate-based chemotherapy and whole brain irradiation $[4,5]$. DNL has been found in immunosuppressed patients, including HIV patients [1]. Single case reports described DNL occurring with infectious diseases and sepsis, suggesting an excessive inflammatory response as etiologic factor [9]. Symptoms occur directly after therapy or many months later [4]. Thus, DNL in our patient may be related to the direct effects of alemtuzumab, e.g. immunosuppression, or it could be linked to side effects of alemtuzumab infusions such as the respiratory tract infection with sepsis or the warm hemolytic anemia and its immunosuppressive treatment. The autopsy showed acute necrotizing lesions, suggesting they developed simultaneously with the sepsis and hemolytic anemia.

Clinical symptoms of DNL include irritability, somnolence, rapidly progressive subcortical dementia and coma with fatal outcome. Neuroimaging may be normal 


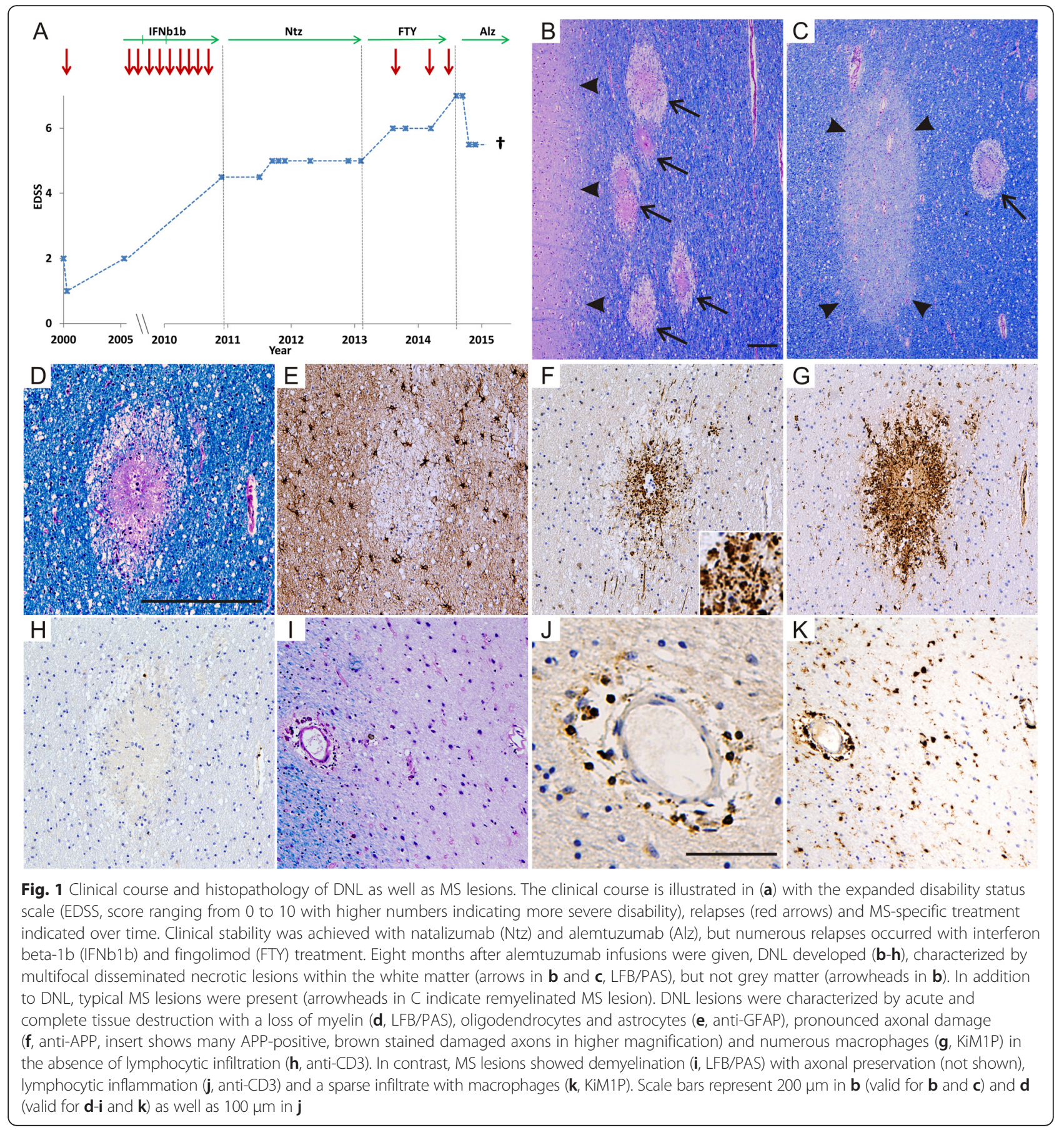

in early stages and show white matter lesions, often calcified, in later stages [4]. The observed loss of consciousness in our patient may have been related to DNL. DNL can be difficult to recognize clinically and is thus easily overlooked.

In conclusion, the clinician should be aware of DNL as a possible direct or indirect side effect of alemtuzumab treatment in MS that is rare but severe, and has never before been described in the literature.

\section{Funding}

None.

\section{Authors' contribution}

Conception and design of the study as well as neuropathological analysis: IM and WB. Patient care: PR and BAK. Medical documentation analysis, drafting of the manuscript: IM. Editing of the manuscript: IM, PR, BAK and WB. All authors read and approved the final manuscript.

\section{Competing interests}

IM reports grants from German Ministry for Education and Research BMBF, "German Competence Network Multiple Sclerosis" (KKNMS), Pattern MS/NMO) 
and Biogen Idec as well as personal fees from Biogen Idec, Bayer Healthcare, TEVA, Serono, Novartis outside the submitted work. PR received honoraria from Biogen, Bayer, Genzyme, Merck, Novartis, Roche and Teva for lectures and advisory board meetings. BAK has received honoraria from Biogen, Genzyme, Merck Serono, Novartis, Roche and Teva for lectures and advisory board meetings. WB has received honoraria for lectures and travel grants by Bayer Vital, Biogen Idec, Merck Serono, Teva Pharma, Genzyme, Sanofi-Aventis and Novartis. He is a member of scientific advisory boards for Teva Pharma, Biogen Idec, Novartis and Genzyme. WB receives research support from Teva Pharma, Biogen Idec, Genzyme and Novartis and serves on the editorial boards of Neuropathology and Applied Neurobiology, Multiple Sclerosis International and Therapeutic Advances in Neurological Disorders. WB is supported by the Deutsche Forschungsgemeinschaft, SFB Transregio 43, "The brain as a target of inflammatory processes", project B9, the German Ministry for Education and Research (BMBF, "German Competence Network Multiple Sclerosis" (KKNMS), Pattern MS/NMO and German MS Brain Bank), and by the Klaus Tschira Foundation for projects other than the submitted work.

\section{Ethics approval and consent to participate}

Study approval was granted by the University Medical Center Göttingen ethical review committee (14/05/03). Research was carried out in compliance with the Helsinki Declaration.

\section{Author details}

'Department of Neuropathology, University Medical Center Göttingen, Georg-August-University, Robert-Koch-Str. 40, 37075 Göttingen, Germany. ${ }^{2}$ Department of Neurology, Sozialstiftung Bamberg Hospital, Buger Str. 80, 96049 Bamberg, Germany. ${ }^{3}$ Neurological Practice Kallmann, Kärntenstr. 2, 96052 Bamberg, Germany.

Received: 25 July 2016 Accepted: 25 July 2016

Published online: 08 August 2016

\section{References}

1. Anders KH, Becker PS, Holden JK, Sharer LR, Cornford ME, Hansen LA, Hamilton R, Vinters HV. Multifocal necrotizing leukoencephalopathy with pontine predilection in immunosuppressed patients: a clinicopathologic review of 16 cases. Hum Pathol. 1993;24:897-904.

2. Coles AJ, Compston DA, Selmaj KW, Lake SL, Moran S, Margolin DH, Norris $\mathrm{K}$, Tandon PK. Alemtuzumab vs. interferon beta-1a in early multiple sclerosis. N Engl J Med. 2008;359:1786-801.

3. Weetman AP. Graves' disease following immune reconstitution or immunomodulatory treatment: should we manage it any differently? Clin Endocrinol (Oxf). 2014;80:629-32. doi:10.1111/cen.12427.

4. Perry A, Schmidt RE. Cancer therapy-associated CNS neuropathology: an update and review of the literature. Acta Neuropathol. 2006;111:197-212. doi:10.1007/s00401-005-0023-y.

5. Rubinstein LJ, Herman MM, Long TF, Wilbur JR. Disseminated necrotizing leukoencephalopathy: a complication of treated central nervous system leukemia and lymphoma. Cancer. 1975:35:291-305.

6. Elimelakh M, Dayton V, Park KS, Gruessner AC, Sutherland D, Howe RB, Reding MT, Eastlund T, van Burik JA, Singleton TP, et al. Red cell aplasia and autoimmune hemolytic anemia following immunosuppression with alemtuzumab, mycophenolate, and daclizumab in pancreas transplant recipients. Haematologica. 2007;92:1029-36.

7. Niscola P, Ragusa D, Scaramucci L, Giovannini M, Piccioni D, Tendas A, Perrotti A, de Fabritiis P, Del Poeta G. Unexplained severe Coombs-negative hemolytic anemia during treatment of refractory chronic lymphocytic leukemia with alemtuzumab. Ann Hematol. 2014;93:863-5. doi:10.1007/ s00277-013-1877-Z

8. Zanella A, Barcellini W. Treatment of autoimmune hemolytic anemias. Haematologica. 2014;99:1547-54. doi:10.3324/haematol.2014.114561.

9. Sharshar T, Gray F, Poron F, Raphael JC, Gajdos P, Annane D. Multifocal necrotizing leukoencephalopathy in septic shock. Crit Care Med. 2002;30:2371-5. doi:10.1097/01.CCM.0000029189.65178.C5. 